TAO, Vol. 15, No. 3, 311-327, September 2004

\title{
Achievements of NASDA's Earthquake Remote Sensing Frontier Project
}

\author{
M. Hayakawa ${ }^{1, *}$, O. A. Molchanov ${ }^{1,2}$ and NASDA / UEC team
}

(Manuscript received 14 April 2004, in final form 23 June 2004)

\begin{abstract}
After the disastrous Kobe earthquake, the former Science and Technology Agency (STA) of the Japanese Government proposed the Integrated Earthquake Frontier Project. Within the framework of this project, two institutions [RIKEN (Physical and Chemical Institute) and NASDA (National Space Development Agency of Japan)] were asked to carry out over five years a feasibility study of the use of electromagnetic phenomena in short-term earthquake prediction. Since 1996 the RIKEN group has been engaged in the study of earth current and related phenomena. Meanwhile, under the NASDA project named "Earthquake Remote Sensing Frontier Project", we performed an overal study of seismo-electromagnetic phenomena occurring not only in the lithosphere, but also in the atmosphere and ionosphere. Our five-year study has yielded that the seismic effect expresses itself not only in the lithosphere [such as by ultra low frequency (ULF) electromagnetic emissions, acoustic emissions etc.], but also in the atmosphere and ionosphere (by means of pre-existing transmitter signals). Most importantly, we find convincing evidence for seismo-ionospheric perturbations on the basis of subionospheric very low frequency (VLF) / low frequency (LF) signals. We have established a new challenging scientific field, "Lithosphere-atmosphere - ionosphere coupling" or "Electromagnetics in the coupled lithosphere-atmosphere- ionosphere system". The study of this coupling would contribute to scientific short-term earthquake prediction. This paper reviews scientific output from our Frontier Project.
\end{abstract}

\footnotetext{
${ }^{1}$ Department of Electronic Engineering, the University of Electro-Communications, 1-5-1 Chofugaoka Chofu, Tokyo, Japan

2 Institute of Physics of the Earth, Russian, Academy of Science, Moscow, Russia

* Corresponding author address: Prof. M. Hayakawa, Department of Electronic Engineering, the University of Electro-Communications, 1-5-1 Chofugaoka Chofu, Tokyo, Japan; E-mail: hayakawa@whistler.ee.uec.ac.jp
} 
(Key words: NASDA's Earthquake Remote Sensing Frontier, Subionospheric VLF waves, Ionospheric perturbations, ULF emissions, Lithosphere-Atmosphere-Ionosphere Coupling)

\section{INTRODUCTION}

There have been many reports on electromagnetic phenomena possibly related to earthquakes (Hayakawa and Fujinawa 1994), however, we think the seismo-electromagnetics field was not well organized world-wide before the 1995 Kobe earthquake. Of course, we were aware of the very important pioneering studies performed in the former USSR, Greece etc.

After the disastrous Kobe earthquake, the Japanese Government through its Science and Technology Agency (STA) conducted the Integrated Earthquake Frontier Project. Two institutions, the Physical and Chemical Institute (RIKEN) and the National Space Development Agency (NASDA) of Japan were asked to undertake a feasibility study of the use of electromagnetic phenomena for short-term earthquake prediction. Hayakawa was asked to be responsible for NASDA's Earthquake Remote Sensing Frontier Project which was carried out over five years starting in 1996.

NASDA and the University of Electro-Communications (UEC) have been engaged in the overall study of electromagnetic phenomena associated with earthquakes. In the beginning of the Frontier Project, we did not know much about what was happening in different areas in association with earthquakes, hence we decided to adopt different kinds of observations: (1) radio sounding; monitoring of the ionospheric perturbations associated with earthquakes by means of VLF/LF subionospheric propagation, (2) satellite observation of plasma perturbations and wave emissions inside the ionosphere, (3) ionospheric density mapping by means of GPS receivers, (4) seismo-atmospheric perturbations by means of radio sounding, (5) subsurface measurement of seismogenic emissions (at ULF/ELF, and VLF) and acoustic emissions and (6) remote sensing of Earth's surface temperature. Our five-year study has yielded that electromagnetic phenomena are taking place not only in the lithosphere, but the seismic effects are also very apparent in the atmosphere and ionosphere. Finally, we have proposed a new scientific field called lithosphere-atmosphere - ionosphere (LAI) coupling or electromagnetic phenomena in the coupled system of lithosphere, atmosphere and ionosphere (Hayakawa 1999; Hayakawa and Molchanov 1999; Hayakawa et al. 2002). This paper is the summary of our NASDA Frontier Project.

\section{SEISMO-IONOSPHERIC PERTURBATION AS DETECTED BY SUBIONOSPHERIC VLF/LF PROPAGATION}

Some time ago Russian colleagues suggested the use of VLF subionospheric signals to study seismo-ionospheric perturbations for some large earthquakes, but we were the first to present convincing results on ionospheric perturbations associated with the Kobe earthquake (on 17 January, 1995) by means of subionospheric VLF propagation [3,4]. By using the same 
analysis procedure (amplitude and phase fluctuations) as our Russian colleagues, we found some increases in such fluctuations in amplitude and phase before the Kobe earthquake (not shown here), but the data were not convincing as a precursor to the earthquake. So, we proposed the method of terminator time. We discovered a significant shift in terminator times as shown in Fig. 1, illustrating the day-to-day sequence of diurnal variations of the phase $(10.2 \mathrm{kHz})$ as measured at Inubo (geographic coordinates; $35^{\circ} 42^{\prime} \mathrm{N}, 140^{\circ} 52^{\prime} \mathrm{E}$ ) using a VLF Omega signal transmitted from Tsushima $\left(34^{\circ} 37^{\prime} \mathrm{N}, 129^{\circ} 27^{\prime} \mathrm{E}\right)$. The terminator time is defined as the time when the diurnal phase (or amplitude) variation exhibits a minimum around sunrise and sunset (which we call morning $(\mathrm{tm})$ and evening (te) terminator times). Figure 1 shows a surprising result: a significant change in terminator times before the quake. The point at the minimum around sunrise is indicated by a black dot, and the time is called $t m$, while, the time with a white dot is called $t e$. The vertical lines indicate $t m$ and $t e$ under normal (unperturbed) conditions, so that the hatched area means the deviation or shift in the terminator time from the corresponding unperturbed situation. Hence, it is clear that $t m$ shifts to early time and te to later time. This effect is also confirmed by analysis over a much longer data length ( \pm 4 months; over a total of eight months) in Fig. 2. The full line indicates terminator time (te, phase) averaged over \pm 1 day on each day ( 3 day running average). The estimation of mean value (0) and standard deviation $(\sigma)$ is based on averaging over \pm 1.5 month period around a particular day. This figure shows the deviation in te from the mean $(0)$, and the $\pm 2 \sigma$ lines are also drawn. This figure indicates that the only significant peak is seen just before the quake. Also, after studying the correlation of this anomalous propagation with other possibly related phenomena (magnetic activity, solar activity, rainfall etc.), we have not found any recognizable correlation with any of those phenomena, so that we conclude that this propagation anomaly is highly likely to be associated with the quake. We have also found the similar tendency for $t_{m}$ (i.e., lead-time).

Hayakawa et al. (1996) and Molchanov et al. (1998) suggested to explain the change in the lower ionosphere by means of the theory of subionospheric VLF propagation over a short distance $(\sim 1,000 \mathrm{~km})$ for which there exist several modes of propagation (i.e., terminator time is the consequence of wave interference of those modes), on the basis of the comparison of theoretical estimations with the experimental data, and from this the authors concluded that the lower ionosphere might have been lowered by a few kilometers.

Encouraged by the results for the Kobe earthquake, Molchanov and Hayakawa (1998) performed the same analysis (terminator times) for a number of large earthquakes (with magnitude greater than 6.0) over a period of 13 years, using the same Inubo data for the Tsushima Omega transmission. They found that, when the earthquakes are shallow (depth less than 50 $\mathrm{km})$ and located very close to a great-circle-path, a large fraction $(\sim 80 \%)$ of the earthquakes investigated shows the propagation anomaly (in the sense of a significant shift in terminator times (i.e., ionospheric perturbations). Another important finding is that when we have the propagation anomaly (ionospheric perturbations) a harmonic analysis on the data of the terminator times exhibit enhanced modulation with periodicities of 5 days or 9-11 days. This implies that atmospheric oscillations with those periodicities may play an important role in the coupling from the lithosphere to the ionosphere. Figure 3 illustrates an example of the en- 


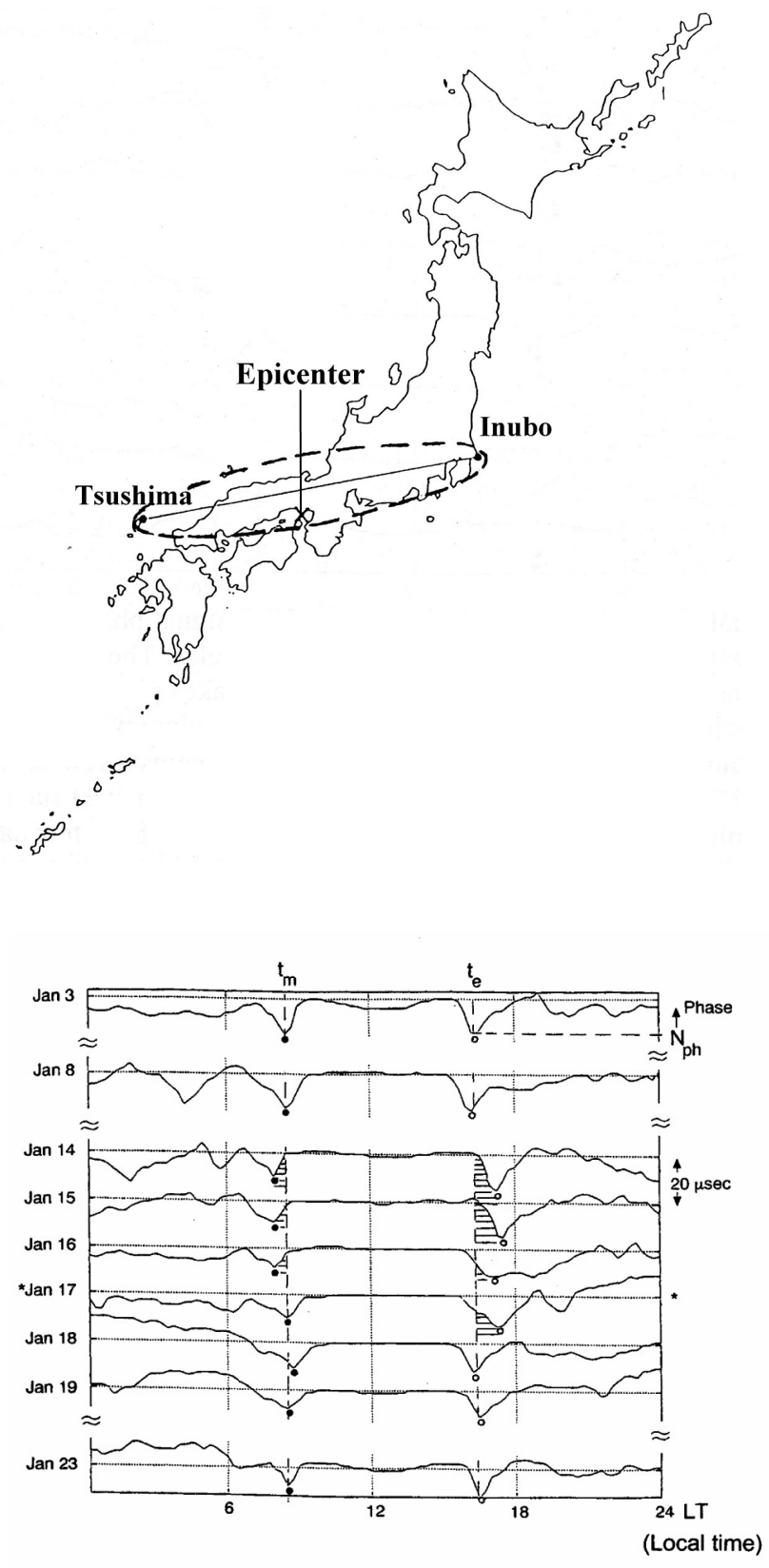

Fig. 1. (Upper panel) Location of the VLF transmitter and receiver (together with the epicenter). (Lower panel) Sequential plot of diurnal variation of phase measurement at Inubo of Tsushima Omega transmission ( $f=10.2$ $\mathrm{kHz}$ ) just around the Kobe earthquake. A significant change is seen in the terminator times. 


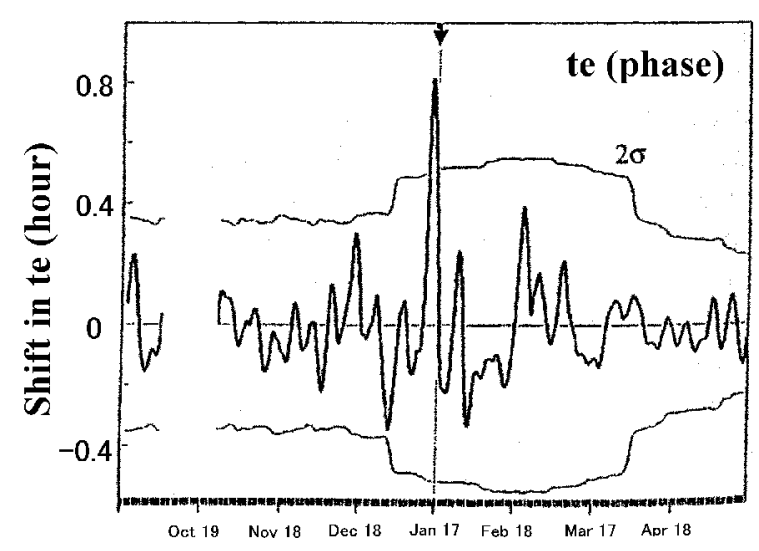

Fig. 2. Change in terminator time for a longer period ( \pm 4 months around the quake). A significant change (exceeding $2 \sigma$ line) is seen only before the quake.

Dynamic periodogram

October 24, 1994 - May 31, 1995

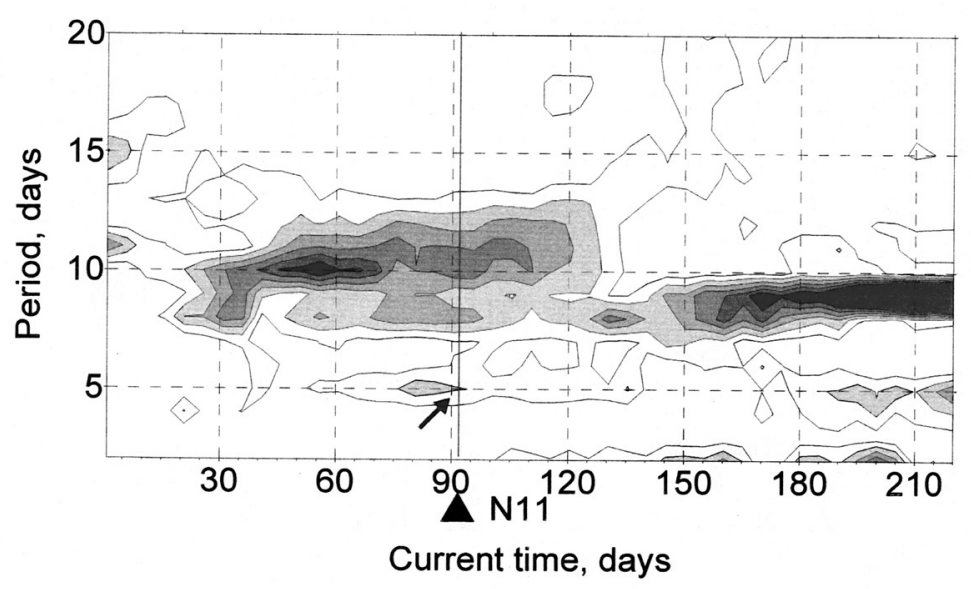

Fig. 3. An example of enhancement of the atmospheric oscillations with periods of 5 days and 9-11 days for the same Kobe quake.

hancement of such atmospheric oscillations before the same Kobe quake. The earthquake date is given by an upward arrow, and you can notice a strong wavelet with a period of $\sim 11$ days and a small, less weak wavelet with a period of 5 days just around the earthquake date. Recently we proposed gravity waves as the carrier because of their stronger tendency of upward propagation in the lithosphere-ionosphere coupling, with the planetary wave as the modulating signal. Based on the study of fluctuation spectra of our observational data (on amplitude and phase), we found an enhanced occurrence of fluctuation power in the frequency range (10 minutes to 2 hours) of gravity 
waves, probably associated with earthquakes (Molchanov et al. 2001). These findings may provide a fundamental basis for the study of LAI coupling.

From the commencement of this Earthquake Remote Sensing Frontier Project in 1996, we installed VLF receiving stations all over Japan. Initially we established two key stations in Chofu (UEC) and in Kasugai, Nagoya (Chubu University), where our new receivers are designed to receive simultaneously 4 5 VLF transmitters such as NWC (Australia), NPM (Hawaii), CHI (China) etc. Year by year we increased the number of receiving stations. Also, faced with the closure of the Omega VLF transmission on 30 September, 1997, we started to pay more attention to the JG2AS (JJY) $(40 \mathrm{kHz})$ transmission (which was moved from its previous place to a new site in Fukushima Prefecture on 10 June, 1999 with an increase in radiation power up to $10 \mathrm{~kW}$ ). Figure 4 illustrates only the VLF receiving network in Japan, and we also receive a $40 \mathrm{kHz}$ signal at those seven VLF stations. Figure 5 illustrates one example of the reception of Tsushima Omega signal $(10.2 \mathrm{kHz})$ observed at Chofu, which is the result of our Frontier Project. In March 1997 (before the closing down) a seismic swarm occurred at Izu peninsula [a dark region in Fig.1 (near Tokyo)], which provided a good opportunity to study a typical correlation between the VLF propagation anomaly (ionospheric perturbations) and seismic activity within the Fresnel zone.

The change in terminator time (te in amplitude) is plotted around the running mean (indicated by a horizontal straight line), together with the $2 \sigma$ line (indicated by the upper thick horizontal line). As for the mean and standard deviation, we have used the average during this March. Earthquakes on each day are indicated as a function of their occurrence time (U.T.), with the size of the bars being proportional to the quake magnitudes. A seismic swarm took place from March 3 to March 10. In what appears to see a good correspondence with this swarm we found a significant shift in terminator time (te, amplitude) during the same period from March 4 to March 7. It is impossible for us to have a one-to-one correspondence between individual quakes and the propagation anomaly. However, when we look at the propagation anomaly and seismic activity as groups, we can conclude that a rather good correlation exists between the two and that the propagation anomaly seems to always precede the swarm. The ionospheric perturbation takes place as an integrated effect of a large number of earthquakes during the swarm.

We have already accumulated a few years of data on the basis of our Frontier VLF/LF network observation, and we are ready to perform a statistical study on the correlation between ionospheric perturbations and quakes for different regions of interest. Also, we are analyzing a few event studies for rather strong quakes; such as the Tottori earthquake of 6 October, 2000, the Shizuoka quake of 3 April, 2001, etc. Some of these results are summarized in Hayakawa et al. (2001). We here show some of our recent findings. In addition to the terminator time analyses, we have analyzed the fluctuation (and fluctuation spectra) in the VLF amplitude and phase. Because we are interested in the significant, possible role of atmospheric oscillations in the lithosphere-ionosphere coupling (a few possibilities will be discussed in the last section). With special reference to the fluctuation in the frequency range of AGW (atmospheric gravity wave) of $\sim 10$ minutes to a few hours, the nightime fluctuation spectra in this frequency range have been examined and we have performed their correlation with earthquakes (Shvets et al. 2002, 2004). Our correlation analysis between this fluctuation 
intensity and earthquakes has indicated maxima in correlation coefficient showing the presence of anomalies in VLF fluctuation preceding the earthquakes by 1-5 days. Case study has shown a possible precursory or/and accompanying VLF anomalies for nine from eleven events with magnitude greater than 5. These results may lend support to the important role of AGW in the lithosphere-ionosphere coupling. Theoretical work on the explanation of terminator time shift in association with an earthquake has been attempted by means of 3D subionospheric modeling (Soloviev et al. 2004). Inclusion of the local ionospheric inhomogeneity associated with earthquakes is found to result in a significant shift in terminator time, which is consistent with the experimental work.

Higher LF $(f=100 \sim 300 \mathrm{kHz})$ subionospheric signals from three stations in Siberia, Russia, have been measured at Kasugai and Chofu as a collaborative work with Italian colleagues. Contrary to the former VLF/LF frequencies these LF frequencies monitor the higher altitude of the lower ionosphere.

The observational network depicted in Fig. 4, will enable us to estimate roughly the region of seismo-ionospheric perturbations on the basis of a comparison of anomalies observed at several stations and for different propagation paths. An active probing of the seismo-ionospheric perturbation is being carried out on the basis of the simultaneous measurement of two horizontal magnetic field components and one vertical electric field component. This direction finding result will be published shortly.

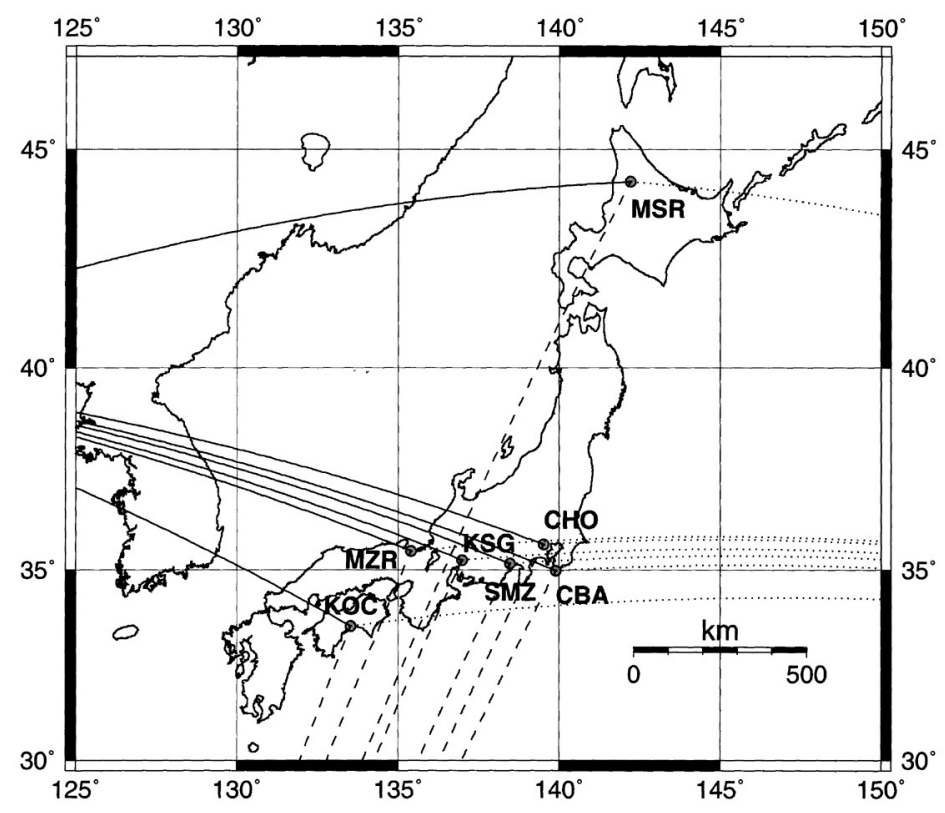

Fig. 4. A network of subionospheric VLF stations (CHO: Chofu; CBA: Chiba; KSG: Kasugai; SMZ; Shimizu; KOC: Kochi; MZR: Maizuru; MSR: Moshiri). 


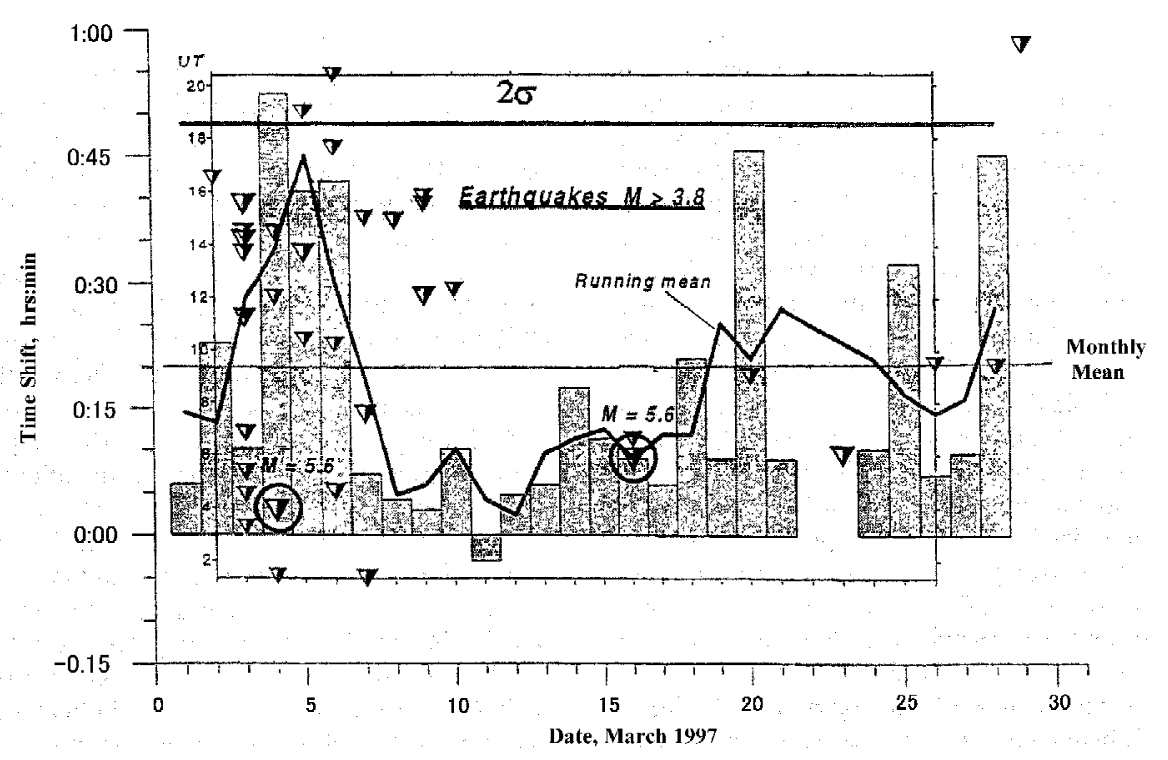

Fig. 5. The result of terminator time variation at Chofu for the Tsushima VLF Omega transmission. The period is March, 1997, when a seismic swarm occurred in the Izu area.

\section{PLASMA DENSITY PERTURBATIONS AND WAVE EMISSIONS IN THE IONOSPHERE}

\subsection{In-site Satellite Observation}

The most important finding of this Frontier Project is that it has demonstrated the probability of some kind of perturbations in the ionosphere in association with earthquakes. VLF/ LF subionospheric signals are known to reflect from the lower D region of the ionosphere, but there may also be some seismo-influence on the upper region of the ionosphere. We have utilized former satellite data for this study, in particular the Russian satellite, Intercosmos 24 was used to investigate the plasma density perturbations associated with earthquakes. After analyzing the data for more than 7,000 hours all over the world during 1989-1990 on board Intercosmos 24, we selected the basic parameters of our recording; geographic latitude, geographic longitude, local time (L.T.), altitude $(\mathrm{H})$, and magnetic index (Kp). By imposing the appropriate boundaries to these parameters, we divided the entire volume of data into abundant elementary cells and calculated the different values of density variations in each elementary cell. We found a reliable correlation between the global distribution of seismic activity and ion density variations (see Fig. 6). The upper panel of Fig. 6 illustrates the longitudinal distribution of the number of earthquakes with magnitude greater than 5 during 1989 and 1990, and the lower curve indicates relative standard deviation values (RNSD) (in full lines) 
which are defined as NSD $/<\mathrm{NSD}>$, where NSD is the normalized standard deviation and $<>$ means the average over all the cells with equal LT, H, and Kp parameters. The best parameters for the correlation with earthquakes are the normalized standard deviation (NSD) and relative normalized standard deviation (RNSD) as given in Fig. 6. Maximum values of ion density NSD correlated with seismic activity are 10-15\% and clear correlation was found only for daytime (10-16h LT) and the altitude range from 500-700 km. Further study has continued together with the study of plasma waves (ELF and VLF). We found that the equatorial anomaly common at low latitude is strongly modified by seismic activity. See the details in Hayakawa et al. (1999).

Using the data of ion density, electron temperature and electric field fluctuations observed aboard the Active (IK-24) satellite, we have tried to find correlation with seismicity. About 50 large earthquakes with magnitude $M \geq 6$ occurred during the observation period, but some correlation with seismicity could be seen only for 4 , all of them in the near-equatorial area. It seems that seismo-induced modification of plasma density near the crests of the equatorial anomaly can appear at altitudes of 500-800 km and intensive turbulence is developed at the poleward slopes of the equatorial anomaly. This phenomenon may be interpreted in terms of the influence of acoustic-gravity waves from a seismic source on the marginally stable equatorial anomaly dynamic structure (Hayakawa and Molchanov 2002).

\subsection{GPS Monitoring}

Another way of monitoring the ionospheric plasma perturbations associated with earthquakes is the use of GPS data. In Japan a large number of GPS receivers have been installed by

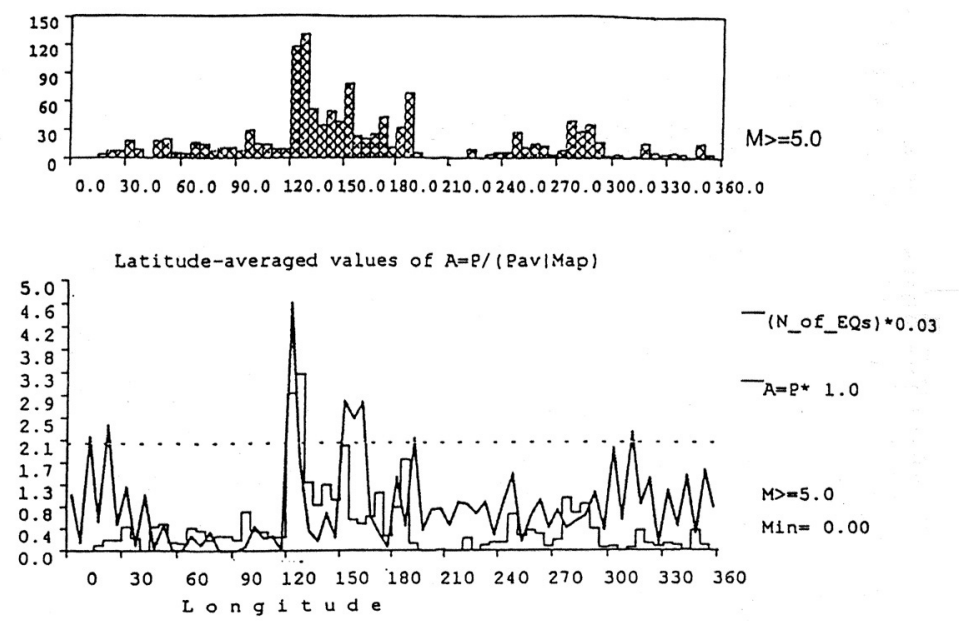

Fig. 6. Longitudinal dependence of the earthquake occurrence rate $(\mathrm{M} \geq 5)$ and the longitudinal variation of the relative normalized standard deviation $(\mathrm{h}=500-1000 \mathrm{~km}), \mathrm{Kp}<3, \mathrm{LT}=10-10 \mathrm{~h}$. 
the Geological Survey of Japan. Their data will be utilized to monitor the ionospheric TEC (total electron contents) profile by the simultaneous use of the GPS data by means of the inversion technique. A group in Taiwan has been performing extensive studies on this subject, and has found convincing evidence of ionospheric F2 layer perturbations, associated with Taiwan earthquakes (Liu et al. 2002). We have already found some anomalies in the TEC for two earthquakes [Geiyo (1999) and Tottori (2002) earthquakes], but it is not so certain that these are really associated with the earthquakes. So that, we are now applying the sophisticated signal processing (like wavelet analysis, Principal component analysis etc.) to make clear whether those anomalies are likely to be associated with earthquakes or not. These results will be compared with the results from Sections 2 and 3.

\section{SEISMO-ATMOSPHERIC PERTURBATION AS REVEALED BY THE OBSERVA- TION OF OVER-HORIZON VHF TRANSMITTER SIGNALS}

Next we show one example of the perturbation of the atmosphere that is probably associated with earthquakes by means of observing over-horizon VHF FM transmitter signals (which belong to the same radio sounding category in Section 2) (Hayakawa and Molchanov 2002; Hayakawa et al. 2001). The observation station is at our university at Chofu, and the VHF transmitter signal is from FM Sendai (located at the city of Sendai, frequency $=77.1 \mathrm{MHz}$, horizontal polarization, power $=5 \mathrm{~kW}$ ). We have adopted a rather sophisticated antenna array system, which enables us to determine the direction (bearing and elevation angles) of the received signal. Each antenna is a 5 -element Yagi, and for the elevation angle $0^{\circ}$ (this means horizontal) there are 3 antennas with different bearing (azimuth) angles $\left(0^{\circ}+30^{\circ}\right.$, and $-30^{\circ}$; $0^{\circ}$ means the azimuth to FM Sendai from UEC, and +(-) indicate the east (west) of the path). Additionally, we have installed three antenna systems with different elevation angles $\left[0^{\circ}\right.$ (horizontal), $45^{\circ}$, and $90^{\circ}$ (vertical upwards)] for the fixed bearing of $0^{\circ}$ (toward the transmitter). These are all for the same horizontal polarization. The last antenna system is for the vertical polarization (bearing $=0^{\circ}$ and elevation $=0^{\circ}$ ). The same frequency of $77.1 \mathrm{MHz}$ is used in Tokyo at the University of Air, so that the observation is limited only to the local time $0 \sim 6 \mathrm{~h}$ after midnight. Under normal conditions, the observed signals for all antenna channels are at background level, but we can occasionally observe significant signal increases, the magnitude of which depends on the direction of arrival. One example is given in Fig. 7. By recording the audio signal we confirmed that the observed signals are from the FM Sendai. Comparing the outputs from different bearings (for elevation $=0^{\circ}$ ) we found that the output from bearing $=$ $30^{\circ}$ is much larger than that for bearing $=-30^{\circ}$, which means that the wave is coming from east of the direct path. Of course, we can determine the bearing as $+20^{\circ}$ from the direction finding (comparison of outputs from $+30^{\circ}$ and $-30^{\circ}$ bearings). Further, the incident angle (or elevation angle) can be estimated from the comparison of the outputs from the antennas with elevation angles $\left(0^{\circ}, 45^{\circ}\right.$ and $\left.90^{\circ}\right)$, which yields an elevation angle of less than $10^{\circ}$. Based on the statistical study for many events during the period of February 1st to 30 June, 2000 including one event in Fig.7, it is found that over-horizon FM signals can be observed on abnormal conditions, which are probably associated with earthquakes. The cross-correlation of the signal reception 
with the earthquakes indicates that the abnormal reception of over-horizon FM signals begins about 7 days before an earthquake, and the directional finding suggests that the transmission is due to favorable conditions in the atmosphere (troposphere or stratosphere) caused by some form of pre-seismic activity (Hayakawa and Molchanov 2002).

\section{LITHOSPHERIC ULF AND AE EMISSIONS}

The initial and boundary conditions for LAI coupling and interaction can be investigated by means of ULF and AE (acoustic emission) observations. These emissions are considered to be manifestation of microfracturing taking place in the focal zone. For example, Molchanov and Hayakawa (1995) proposed a mechanism of seismogenic ULF emissions in terms of microfracturing. In addition to the famous two ULF events (Spitak and Loma Prieta), a ULF signature was reported for the Guam earthquake (Hayakawa et al. 1996), further supported by sophisticated signal analysis (Hayakawa et al. 1999), named fractal analysis.
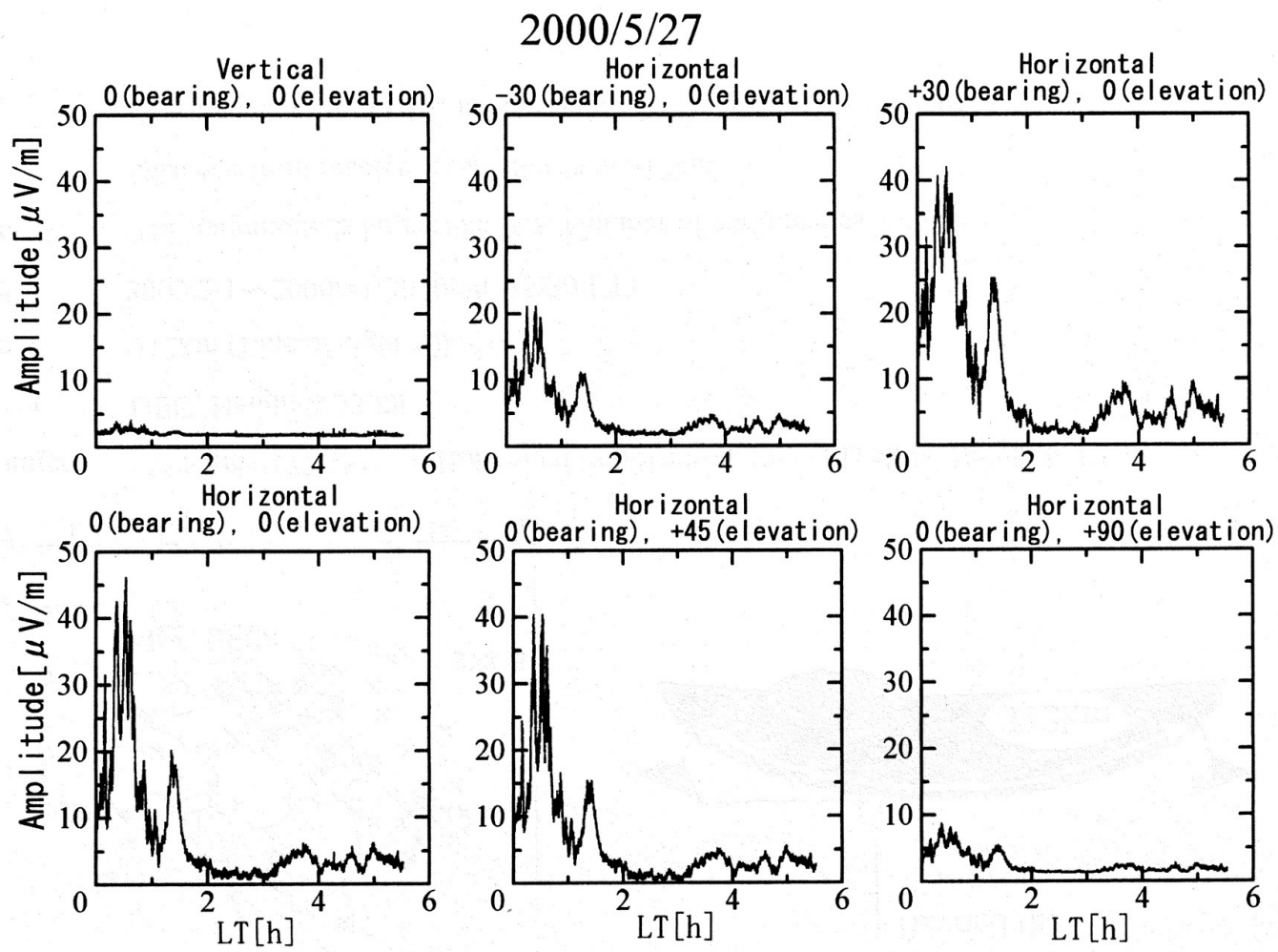

Fig. 7. An example of the reception of over-horizon FM transmitter signals at UEC on 27 May, 2000. The outputs from different antenna systems are indicated. 
Additional convincing evidence on the appearance of ULF emissions before a large earthquake has been obtained by Hayakawa et al. (2000) for the quake at Biak, Indonesia in February, 1996.

Within the framework of NASDA's Frontier Project, we installed a network of ULF measurements in the Kanto area: (1) Izu, (2) Chiba, (3) Kakioka, (4) Chichibu, and (5) Matsushiro. Especially, in the Izu and Chiba areas, we have established a differential array by using 3-4 closely spaced (spacing, 5-10 km) ULF stations, which are useful to increase the S/N ratio for seismogenic emissions and can also help in direction finding. One example of ULF emissions associated with the seismic swarm of March-May 1998 has been obtained by means of our developed polarization measurements. Some results on a precursory signature for the Izu islands earthquakes in July 2000 were obtained by using a sophisticated signal processing (such as principal component analysis) of the simultaneous ULF data observed at three stations on the Izu peninsula (Hayakawa et al. 2001). Here we present interesting results for the Izu islands earthquakes of July 2000 (Gotoh et al. 2002). Figure 8 shows the Izu ULF stations and the epicenters of several rather large earthquakes. The distance between our ULF stations and the epicenters is about $80 \sim 100 \mathrm{~km}$, a critical distance for the ULF detection. Three closely spaced stations enable us to perform the principal component analysis. We can distinguish three possible noise sources. The first principal component is found to reflect the terrestrial geomagnetic effect. The second one refers to human activity because we observe a typical diurnal pattern with a lunchtime effect. The third and smallest component is the residual, in which we can expect the presence of any seismic influence. Figure 9 shows the temporal evolution of the third principal component $\left(\lambda_{3}\right)$ at a frequency of $10 \mathrm{mHz}$. This component is seen to exhibit an increase from May (about two months before the quake), this increase lasts for $\sim 1.5$ months and is followed by a significant quiet period about one week before the quake. We also find a significant increase a few days before each of the quakes. This kind of temporal

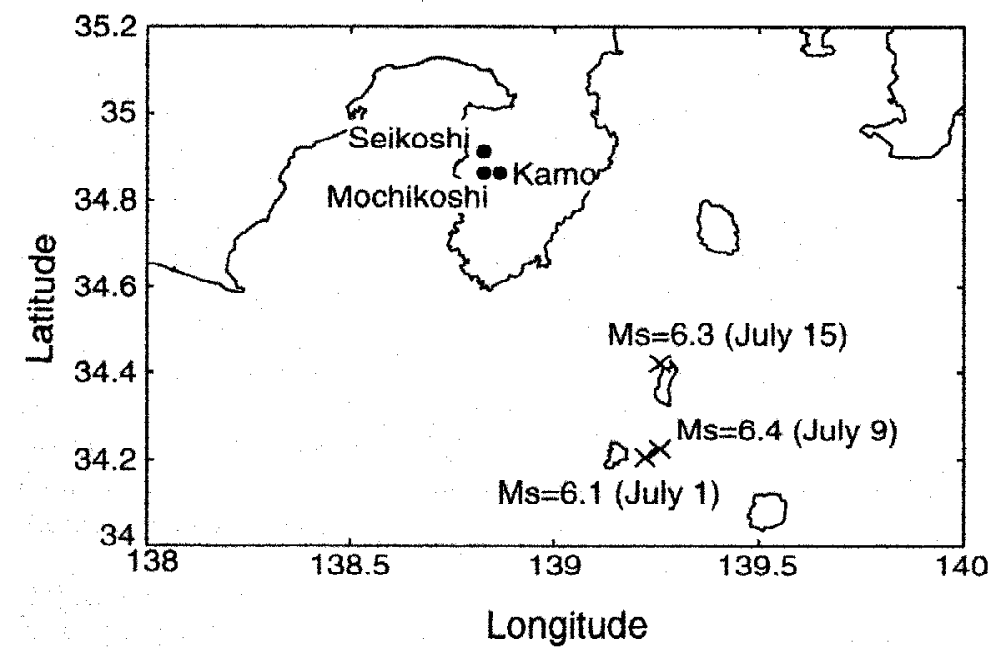

Fig. 8. Relative location of Izu ULF stations and epicenters of the Izu islands earthquakes. 
evolution is very similar to that observed prior to the Loma Prieta and other quakes, so that this is highly likely to be an earthquake-related ULF signature. However, we wait for a rather large earthquake close to any one of our stations.

We published our pioneering paper on the ULF generation by microfracturing (Molchanov and Hayakawa 1995), e.g., the creation and relaxation of charges on the walls of opening cracks in the earthquake hypocenter as a possible cause of the electromagnetic noise prior to an earthquake. Another approach was suggested, based on the same microfracturing, where the electromagnetic perturbation is caused by the MHD effect from a propagating seismic wave. Inductive seismo-electromagnetics ULF emission has been proposed, probably suitable for the co-seismic ULF emission [see our papers in Hayakawa and Molchanov (2002)].

The measurement of AE was started a few years ago at Matsushiro, where many sophisticated, highly sensitive sensors for crustal movement have been installed. Consequently, we proposed a collaboration between our electromagnetic (ULF) group with the AE group in order to bridge the gap between radiophysicists and seismologists. Attempts to find AE associated with earthquakes have been undertaken for some years, and an anomaly in AE in the $800-1200 \mathrm{~Hz}$ range was recorded about 16 hours before the $\mathrm{M}=7.0$ Spitak earthquake, Armenia, 1988, at a distance of $80 \mathrm{~km}$ from the epicenter. However, the conclusions reached from that observation were not completely convincing due to extremely weak signals, with their amplitude spectrum sharply decreasing with frequency. We reported on the distinct intensification of the AE signals associated with EQs as recorded in a tunnel at a depth of about 100 meters at Matsushiro (Gorbatikov et al. 2000). We used special receivers with magneto-elastic detectors, the sensitivity of which increases as cube of frequency. The AE intensification has been verified at four frequency bands, namely $30,160,500$ and $1000 \mathrm{~Hz}$ for several earthquakes in the surrounding area; earthquakes with $\mathrm{M} \sim 3-5$, distance $\sim 20-150 \mathrm{~km}$. It was also found that the increase in $\mathrm{AE}$ activity started about 12 hours before the quakes and decreased after the quakes in the manner as shown in Fig.10. The observations currently continue at Matsushiro. In addi-

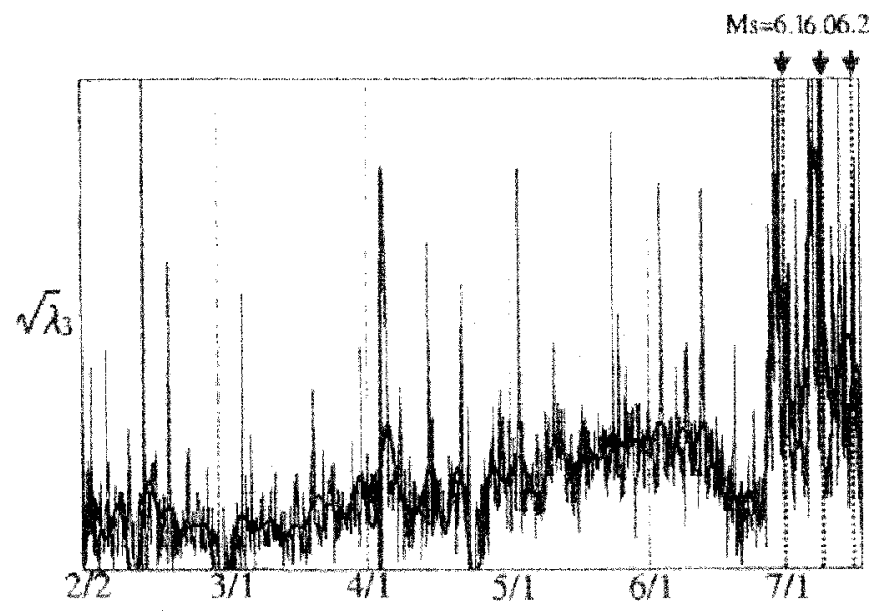

Fig. 9. Temporal evolution of the third principal component at $\mathrm{f}=10 \mathrm{mHz}$ on the basis of a principal component analysis. 
tion we have installed two more AE sites near Matsushiro in order to form a differential array to estimate the direction of arrival.

\section{SURFACE TEMPERATURE MEASUREMENT FROM SATELLITES}

NOAA/AVHRR satellite thermal images indicated the presence of positive thermal anomalies associated with large linear structures and fault systems in the earth's crust. A relationship between thermal anomalies and seismic activity was established for Middle Asia on the basis of a 7-year series of thermal images. In China a thermal anomaly has been identified near Beijing, at the boundary between the mountains and the plain. The size of this anomaly is about $700 \mathrm{~km}$ in length and $50 \mathrm{~km}$ in width. The anomaly appeared about $6-24$ days before and continued for about a week after a major earthquake. The anomaly was sensitive to crustal earthquakes with magnitudes greater than 4.7 and for distances up to $1000 \mathrm{~km}$ and its amplitude was about $3^{\circ} \mathrm{C}$. Thermal anomalies in Japan are found to have more complex shape. The analysis of digital images for Japan shows the following characteristics: (a) the anomaly appears 7 - 10 days before a shock, (b) the anomalies in Japan cover a small area, (c) probably, a thermal anomaly region is located in the Kanto area with a magnitude of up to $6^{\circ} \mathrm{C}$, (d) the tectonic position of the anomalies is still not clear. Understanding these thermal anomalies would be of fundamental interest as an indicator for the seismo-atmospheric and -ionospheric coupling to be discussed in the next section (Hayakawa et al. 2001; Tronin et al. 2002).

\section{LITHOSPHERE-ATMOSPHERE-IONOSPHERE COUPLING MECHANISM}

The most important contribution of this NASDA's Frontier Project is the accumulated evidence of ionospheric perturbations associated with earthquakes and some findings on seismoatmospheric effects, in addition to the already-existing many results on lithospheric phenomena as revealed by subsurface measurements. This leads to a new science field such as "Lithosphere-atmosphere-ionosphere coupling", or "Electromagnetic phenomena in the coupled lithosphere-atmosphere-ionosphere system", and large amount of effort is now being devoted to understanding more fully the mechanisms of this coupling. Figure 11 illustrates the possible channels we suggest for the LAI coupling. The first one is the chemical channel, the 2nd one, the acoustic channel and the 3rd one, the electromagnetic channel. As for the chemical channel, the change in geochemical parameters (gas or radon emanation, water elevation etc.) leads to changes in the air or in conductivity of the air and results in a modification of the atmospheric electric field. This field will then influence the plasma density in the ionosphere. As for the acoustic channel, we may except excitation of atmospheric oscillations, which propagate upward into the ionosphere, modifying the ionospheric plasma density. As for the electromagnetic channel, we know that ULF emissions are generated before an earthquake and those emissions propagate into the inner magnetosphere and they interact with energetic particles there. Then, we expect the precipitation of those particles into the lower ionosphere. Or, we may expect the direct heating (and/or ionization) of the ionosphere by means of seismo-ELF/ VLF waves. We believe a the better understanding of this coupling mechanism will lead to possible short-term earthquake prediction in the future. 


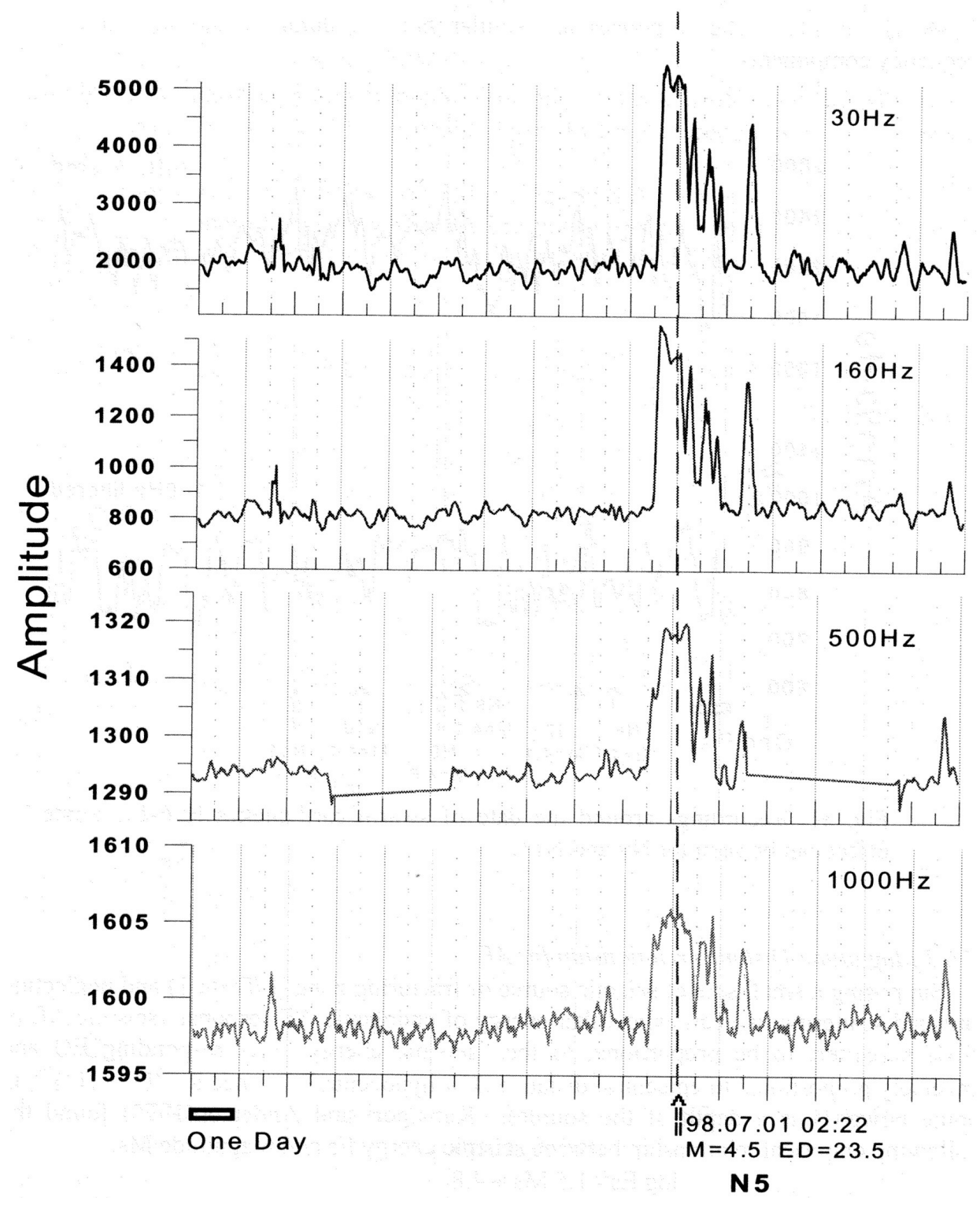

Fig. 10. Recording of $\mathrm{AE}$ at Matsushiro for an earthquake $(\mathrm{M}=4.5$, distance $=$ $23.5 \mathrm{~km})$ on 1 July, 1998.

Acknowledgements The authors are grateful to STA (Science and Technology Agency of Japan) and NASDA for their support to this Frontier Project, and this summary is based on the activity by NASDA/UEC Frontier team. This team included T. Kodama, M. Hirari, A. V. Shvets, Y. Hobara, A. A. Tronin, N. Yamamoto, T. Itoh, K. Miyaki, Y. Fukumoto, K. Akinaga, 


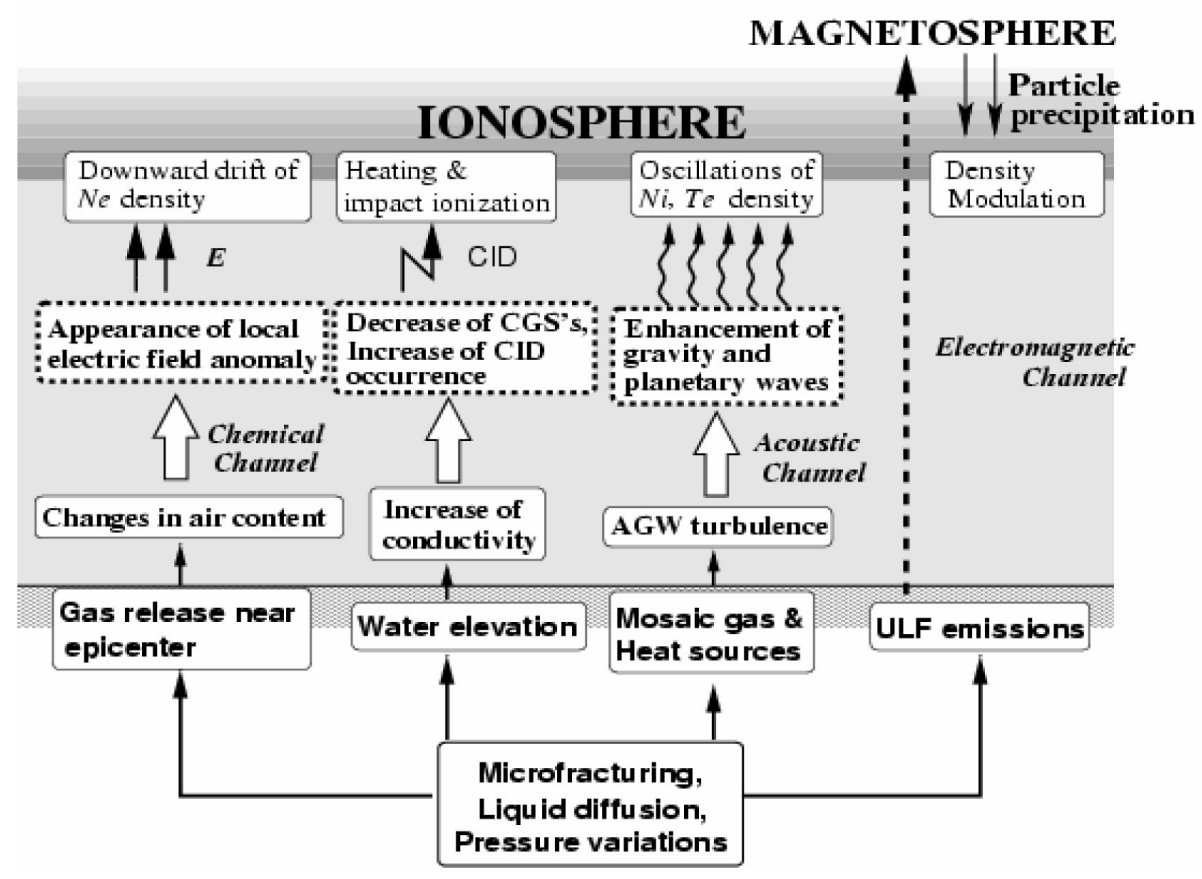

Fig. 11. Possible channels of the Lithosphere-Atmosphere-Ionosphere (LAI) coupling.

and K. Gotoh. Some of the works described in this paper are the result of collaboration with some other (both domestic and foreign) institutions, and we are grateful to the RIKEN group (including S. Uyeda, T. Nagao, K. Hattori) for the collaboration in the fields of ULF emissions and AE. Finally, one of the authors (M.H.) is grateful to Int'l Communications Foundation, The Japan Society of Promotion of Science (JSPS) (\#15403012), The Mitsubishi Foundation, and Hōsō - Bunka Foundation for their support.

\section{REFERENCES}

Gotoh, K., Y. Akinaga, M. Hayakawa, and K. Hattori, 2002 : Principal component analysis of ULF geomagnetic data for Izu islands earthquakes in July 2000. J. Atmos. Electr., 22, $1-12$.

Gorbatikov, A., O. A. Molchanov, M. Hayakawa, S. Uyeda, K. Hattori, T. Nagao, and A. Nikolaev, 2000: Acoustic emission response to earthquake process. Zishin J., 30, 56-63.

Hayakawa, M., and Y. Fujinawa, (Eds.), 1994 : Electromagnetic Phenomena Related to Earth- 
quake Prediction. TERRAPUB, Tokyo, 667p.

Hayakawa, M., (Ed.), 1999: Atmospheric and Ionospheric Electromagnetic Phenomena Associated with Earthquakes, TERRAPUB, Tokyo, 997p.

Hayakawa, M., and O. A. Molchanov, (Eds.), 2002: Seismo-Electromagnetics : Lithosphere, Atmosphere-Ionosphere Coupling. TERRAPUB, Tokyo, 477p.

Hayakawa, M., et al., 2001: NASDA's Earthquake Remote Sensing Frontier Research: SeismoElectromagnetic Phenomena in the Lithosphere, Atmosphere and Ionosphere. Final Report. Univ. of Electro-Communications, 228p.

Hayakawa, M., O. A. Molchanov, T. Ondoh, and E. Kawai, 1996: The precursory signature effect of the Kobe earthquake on subionospheric VLF signals. J. Comm. Res. Lab., 43, 169-180.

Hayakawa, M., T. Itoh, and N. Smirnova, 1999: Fractal analysis of ULF geomagnetic data associated with the Guam earthquake on August 8, 1993. Geophys. Res. Lett., 26, 27992800.

Hayakawa, M., T. Itoh, K. Hattori, and K. Yumoto, 2000: ULF electromagnetic precursors for an earthquake at Biak, Indonesia on February 17, 1996. Geophys. Res. Lett., 27, 1531-1534.

Hayakwa, M., R. Kawate, O. A. Molchanov, and K. Yumoto, 1996: Results of ultra-lowfrequency magnetic field measurements during of the Guam earthquake on 8 August 1993. Geophys. Res. Lett., 23, 241-244.

Liu, J. Y., Y. J. Chuo, S. A. Pulinets, H. F. Tsai, and X. Zeng, 2002: A study on the TEC perturbations prior to the Rei-Li, Chi-Chi and Chia-Yi earthquakes. in Ref. [3], p.297301.

Molchanov, O. A., M. Hayakawa, T. Ondoh, and E. Kawai, 1998: Precursory effects in the subionospheric VLF signals for the Kobe earthquake. Phys. Earth Planet. Inter., 105, 239-248.

Molchanov, O. A., and M. Hayakawa, 1998: Subionospheic VLF signal perturbations possibly related to earthquakes. J. Geophys. Res., 103, 17489-17504.

Molchanov, O. A., M. Hayakawa, and K. Miyaki, 2001: VLF/LF sounding of the lower ionosphere to study the role of atmospheric oscillations in the lithosphere-ionosphere coupling. Adv. Polar Upper Atmos. Res., 15, 146-158.

Molchanov, O. A., and M. Hayakawa, 1995: Generation of ULF electromagnetic emissions by microfracturing. Geophys. Res. Lett., 22, 3091-3094.

Shvets, A. V., M. Hayakawa, and O. A. Molchanov, 2002: Subionospheric VLF monitoring for earthquake-related ionospheric perturbations. J. Atmos. Electr., 22, 87-99.

Shvets, A. V., M. Hayakawa, and O. A. Molchanov, 2004: Study of ionospheric response to regional seismic activity by VLF radio sounding, Special Issue on Seismo Electromagnetics and Related Phenomena. Phys. Chem. Earth, 29, 627-637.

Soloviev, O. V., M. Hayakawa, V. I. Ivanov, and O. A. Molchanov, 2004: Seismo-electromagnetic phenomenon in the atmosphere in terms of 3D subionospheric radio wave propagation problem. Phys. Chem. Earth., 29, 639-647.

Tronin, A. A., M. Hayakawa, and O. A. Molchanov, 2002: Thermal IR satellite data application for earthquake research in Japan and China. J. Geodynamics, 33, 319-534. 
